\title{
Hypercoagulability and Mortality of Patients with Stroke and Active Cancer: The OASIS-CANCER Study
}

\author{
Mi Ji Lee, ${ }^{\mathrm{a}}$ Jong-Won Chung, ${ }^{\mathrm{a}}$ Myung-Ju Ahn, ${ }^{\mathrm{b}}$ Seonwoo Kim, ${ }^{\mathrm{c}}$ Jin Myoung Seok, ${ }^{\mathrm{a}}$ Hye Min Jang, ${ }^{\mathrm{a}}$

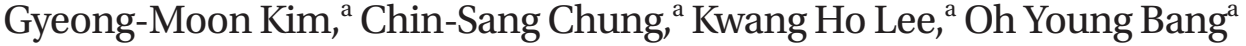 \\ Departments of ${ }^{a}$ Neurology and ${ }^{b}$ Medicine, Samsung Medical Center, Sungkyunkwan University School of Medicine, Seoul, Korea \\ 'Biostatistics Team, Samsung Biomedical Research Institute, Samsung Medical Center, Seoul, Korea
}

Background and Purpose Patients with active cancer are at an increased risk for stroke. Hypercoagulability plays an important role in cancer-related stroke. We aimed to test whether 1) hypercoagulability is a predictor of survival, and 2) correction of the hypercoagulable state leads to better survival in patients with stroke and active cancer.

Methods We recruited consecutive patients with acute ischemic stroke and active systemic cancer between January 2006 and July 2015. Hypercoagulability was assessed using plasma D-dimer levels before and after 7 days of anticoagulation treatment. The study outcomes included overall and 1-year survival. Plasma D-dimer levels before and after treatment were tested in univariate and multivariate Cox regression models. We controlled for systemic metastasis, stroke mechanism, age, stroke severity, primary cancer type, histology, and atrial fibrillation using the forward stepwise method.

Results A total of 268 patients were included in the analysis. Patients with high (3rd-4th quartiles) pre-treatment plasma D-dimer levels showed decreased overall and 1-year survival (adjusted HR, 2.19 [95\% Cl, 1.46-3.31] and 2.70 [1.68-4.35], respectively). After anticoagulation treatment, posttreatment D-dimer level was significantly reduced and independently associated with poor 1-year survival (adjusted HR, $1.03[95 \% \mathrm{Cl}, 1.01-1.05]$ per $1 \mu \mathrm{g} / \mathrm{mL}$ increase, $P=0.015)$. The successful correction of hypercoagulability was a protective factor for 1-year survival (adjusted HR 0.26 [Cl 0.100.68], $P=0.006)$.

Conclusions Hypercoagulability is associated with poor survival after stroke in patients with active cancer. Effective correction of hypercoagulability may play a protective role for survival in these patients.

Keywords Ischemic stroke; cancer; hypercoagulability; survival; prognosis
Correspondence: Oh Young Bang Department of Neurology, Samsung Medical Center, Sungkyunkwan University, 81 Irwon-ro, Gangnam-gu, Seoul 06351, Korea

Tel: +82-2-3410-3599

Fax: +82-2-3410-0052

E-mail: ohyoung.bang@samsung.com

Received: June 20, 2016

Revised: August 25, 2016

Accepted: October 4, 2016

This study was supported by the Korean Health Technology RCtD Project, Ministry of Health \& Welfare, Republic of Korea (HI13C1521).

The authors have no financial conflicts of interest.

\section{Introduction}

Systemic cancer is associated with an increased risk of ischemic stroke. ${ }^{1,2}$ Active cancer increases the short-term risk of stroke, and ischemic stroke can be the first manifestation of systemic cancer., ${ }^{2,3}$ Characteristics of stroke in patients with active cancer are distinct from those in patients without cancer; for example, embolic pattern and cryptogenic etiology are more frequently observed. ${ }^{1.4}$ Cancer-related hypercoagulability seems to play an important role in the development of cancer-related stroke. ${ }^{5-7}$

Overall survival after stroke is poor in patients with cancer. ${ }^{1}$ Unlike conventional predictors for death in conventional stroke, ${ }_{1}^{8}$ cryptogenic mechanisms, lung cancer, and systemic metastasis 
are reported to be associated with poor survival in cancer-related stroke. ${ }^{1,9}$ Each of those factors is linked to hypercoagulability. ${ }^{3,10}$ If hypercoagulability is associated with increased mortality in cancer-related stroke, it is also of interest to investigate whether survival can be modified with effective antithrombotic treatment. Anticoagulation is associated with improved survival in patients with cancer and deep venous thrombosis. ${ }^{11-15} \mathrm{Howev}-$ er, there is a lack of evidence for the success of antithrombotic therapy in ischemic stroke. Furthermore, whether or not a successful correction of the hypercoagulable state can improve stroke survival has not been investigated.

We hypothesized that cancer-related hypercoagulability and its correction determines survival after stroke in patients with active cancer. We tested whether the level of pre-treatment plasma D-dimer, a surrogate marker of hypercoagulability, is associated with survival. In addition, we tested the plasma D-dimer levels after anticoagulation treatment to evaluate whether the successful correction of hypercoagulability can affect patient survival.

\section{Methods}

\section{Patients}

This is a part of the Optimal Anticoagulation Strategy In Stroke related to Cancer (OASIS-Cancer) study. The OASIS-Cancer study is an observational study to investigate the biological markers for intravascular coagulopathy causing stroke and for monitoring the effects of anticoagulation therapy in patients with active cancer and stroke (ClinicalTrials.gov identifier NCT02743052).

We recruited consecutive patients with acute ischemic stroke and active systemic cancer between January 2006 and May 2015 at the Samsung Medical Center, which is a university hospital with comprehensive stroke and cancer centers. During the study period, patients who 1) had acute ischemic stroke documented based on diffusion-weighted imaging within 7 days after symptom onset, 2) were admitted to or sought consultation at the neurology department for acute stroke, and 3) had known or newly diagnosed active systemic cancer at the time of stroke diagnosis or during hospitalization, were prospectively coded in our cancer-stroke registry. Active cancer was defined as a diagnosis of or treatment for cancer during the 6 months preceding the stroke diagnosis, or the presence of recurrent or metastatic cancer.' Local basal cell or squamous cell carcinoma of the skin and primary CNS tumors were not regarded as systemic cancers and were excluded. In case of newly diagnosed cancer, evaluations for tissue diagnosis and staging were performed by our oncologists. The institutional review board of the Samsung Medical Center approved this study. All participants provided written informed consent before participation.

\section{Work-up for stroke}

The age, sex, National Institutes of Health Stroke Scale (NIHSS) score, and stroke risk factors including hypertension, diabetes mellitus, hyperlipidemia, atrial fibrillation, ischemic heart disease, and tobacco use were obtained for all the patients. The type of primary cancer, histology, and the presence of systemic metastasis were also recorded. Routine laboratory data were collected for all patients. Routine evaluations included electrocardiography, 24-hour Holter monitoring or 72-hour in-patient telemonitoring, echocardiography, brain magnetic resonance (MR) imaging, and MR angiography. Stroke mechanisms were determined using the Causative Classification System (CCS) in a weekly consensus meeting. Conventional stroke mechanisms included large artery atherosclerosis, cardioembolism, small-artery occlusion, and other evident mechanisms. In the absence of conventional stroke mechanisms, the mechanism was classified as cryptogenic.

\section{Hypercoagulability measurements}

Coagulation studies were performed as a routine protocol for acute stroke. Prothrombin time (PT) (s), activated partial thromboplastin time $(\mathrm{s})$, fibrinogen $(\mathrm{mg} / \mathrm{dL})$, and D-dimer levels $(\mu \mathrm{g} /$ $\mathrm{mL}$ ) were measured in patients with acute neurologic symptoms at their arrival in the emergency department. For in-hospital strokes, the coagulation studies were conducted immediately before the computed tomography (CT) testing. The D-dimer level was measured per protocol before antithrombotic treatment in all but five patients.

Antithrombotic treatment was determined based on the stroke mechanism. In patients with increased levels of coagulation markers without conventional stroke mechanisms, anticoagulation treatment was considered as the first-line antithrombotic treatment in the absence of obvious contraindications. Low-molecular-weight heparin (1 unit/kg twice a day) or adjusted-dose warfarin were used. During hospitalization, plasma D-dimer level was serially monitored after the start of the anticoagulation treatment to check whether it had normalized. In this study, the post-treatment D-dimer level measured at $7( \pm 3)$ days after anticoagulation was used to determine successful correction of hypercoagulability. The operational definition of 7 days was used because, after the first week, the continuation of antithrombotic agents and monitoring of D-dimer levels were highly influenced by patients' clinical situations, including invasive procedures performed for tissue diagnosis, hematological abnormalities (e.g., thrombocytopenia), bleeding episodes, or refusal of further treatment. We set the cutoff level of post-treatment plasma D-dimer concentration at $<3 \mu \mathrm{g} / \mathrm{mL}$ to define successful correction, which implies a shift from severe to non-severe hypercoagulability. ${ }^{16}$

After the acute stroke setting, subjects who were regularly fol- 
lowed at our stroke outpatient clinic underwent serial plasma Ddimer level measurements at each outpatient visit. In this study, the outpatient plasma D-dimer levels were determined at 1month ( \pm 15 days) intervals after onset.

\section{Outcome}

Survival status and death date were identified at June 1, 2016 by using data from the Korean National Health Insurance Service database. In Korea, medical insurance is provided to everyone under the National Health System. ${ }^{17}$ In our cohort, information on the survival or death was complete in all except three patients who had foreign nationality. Overall survival was defined as the time from the stroke onset to the date of death as a result of any cause. To determine the overall survival of the surviving patients, survival time was censored at June 1, 2016. To avoid bias due to different follow-up periods, the length of survival was also censored at 1 year after stroke onset (1-year survival). Both overall and 1-year survival were investigated as the outcomes of this study.

\section{Statistical analysis}

The pre-treatment plasma D-dimer levels were divided into quartiles. Demographics, stroke characteristics, and cancer characteristics were compared among quartiles of pre-treatment Ddimer levels with the chi-square test, the Fisher's exact test, or the Kruskal-Wallis test. Mortality rates, median survivals, and interquartile ranges were displayed using the Kaplan Meier method and survival curves were compared between the predefined prognostic factor groups with the log-rank test. A univariate Cox regression model was applied to each predictor of survival, while multivariate Cox regression was performed with a forward stepwise method using quartiles of plasma D-dimer concentrations, systemic metastasis, stroke mechanism, age, NIHSS score, primary cancer type, cancer histology (adenocarcinoma vs others), and atrial fibrillation. Among the primary cancer types, the most significant ones based on the log-rank test were selected as covariates in the multivariate model.

In patients with high (3rd and 4th quartiles) D-dimer levels who underwent anticoagulation therapy, the post-treatment Ddimer levels were tested using the Cox regression model for their overall and 1-year survival after stroke. Post-treatment plasma D-dimer levels were tested as both continuous and categorical variables ( $>3$ vs. $<3 \mu \mathrm{g} / \mathrm{mL}$ ). In order to account for the possible influence of co-existing disseminated intravascular coagulopathy (DIC), subscores of the International Society on Thrombosis and Hemostasis (ISTH) scoring system, ${ }^{16}$ which consisted of platelet count $\left(>100,50-100,<50 \times 10^{3} / \mu \mathrm{L}\right)$, prothrombin time $(<3$, $3-6,>6$ seconds), and fibrinogen level (> 100 vs. $<100 \mathrm{mg} / \mathrm{dL}$ ), were adjusted for with a forward stepwise method as well as by using the aforementioned parameters as covariates in the model. An interaction term between pre-treatment and post-treatment D-dimer levels was added in the Cox regression model to test the post-treatment modification of the relationship between hypercoagulability and survival.

For each Cox regression model, proportional hazard assumptions were examined by testing Schoenfeld residuals for continuous variables and by visual examination of the log (minus log) curves for categorical variables. We used the variance inflation factor to assess the multicollinearity among variables in all the multivariate models. Results are reported as the hazard ratio (HR) with the 95\% confidence interval (CI).

Plasma D-dimer levels collected in the outpatient clinic were tested for their association with mortality in the next month by using logistic regression analysis. The diagnostic value of D-dimer levels measured at any time in predicting death within the next month was determined with the receiver operating characteristic (ROC) curve. The Youden's index (sensitivity+specificity-1) was used to determine the optimal cutoff for the prediction of death within the next month.

All statistical analyses were performed using commercially available software (SPSS for Windows, version 18.0; IBM Corp., Armonk, NY). A $P$ value $<0.05$ was considered significant. Bonferroni correction was performed to correct for multiple comparisons, if any.

\section{Results}

\section{Patients}

The study profile is shown in Figure 1. Of the 4,725 patients in the acute stroke registry during the study period, 271 (5.7\%) were coded as having active cancer at the time of stroke onset. Among them, 268 patients had complete data on survival and death dates (Table 1). The median overall survival was 109 (interquartile range [IOR] 47-468) days. A total 224 of deaths were identified with a median (IQR) survival of 80 (36-209) days. The median (IOR) follow-up for 44 patients alive at the time of censoring was $2,302(521-2,866)$ days. The mortality rate was $18.3 \%$ $(47 / 268)$ at 1 month, $44.4 \%(117 / 268)$ at 3 months, 60.1\% $(159 / 268)$ at 6 months, and $71.6 \%(192 / 268)$ at 1 year.

\section{Baseline characteristics}

In 263 patients with D-dimer measurements obtained per protocol, the median pre-treatment plasma D-dimer level was $9.06 \mu \mathrm{g} / \mathrm{mL}$ (range $0.22-60.00 \mu \mathrm{g} / \mathrm{mL}$ ). The quartiles of the pretreatment D-dimer concentrations were determined: $<2.08 \mu \mathrm{g} /$ $\mathrm{mL}$ (1st quartile), 2.08-9.06 $\mu \mathrm{g} / \mathrm{mL}$ (2nd quartile), 9.06-23.26 


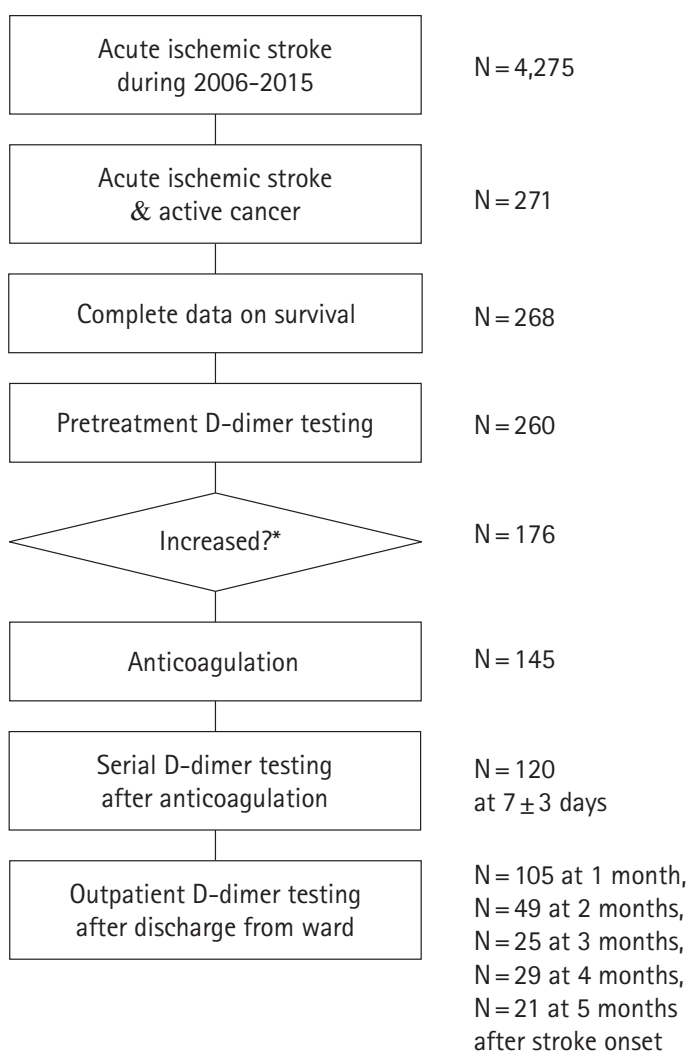

Figure 1. Study profile. *Hypercoagulability and anticoagulation treatment were determined with a consideration of clinical situations. In this frequency analysis, $>3 \mu \mathrm{g} / \mathrm{mL}$ was used as the cutoff of increased plasma D-dimer level.

$\mu \mathrm{g} / \mathrm{mL}$ (3rd quartile), and $>23.26 \mu \mathrm{g} / \mathrm{mL}$ (4th quartile). Patient characteristics according to plasma D-dimer groups are described in Table 1. Patients with the lowest D-dimer levels (1st quartile) had more conventional stroke mechanisms and conventional risk factors such as male sex, dyslipidemia, and atrial fibrillation. In contrast, patients with higher D-dimer levels (3rd4th quartiles) had more adenocarcinoma histology and systemic metastases, and subsequent anticoagulation therapy. Pancreatic and hepatobiliary cancers were less prevalent in patients with the lowest D-dimer levels (1st quartile). Pre-stroke anticoagulation treatment was not associated with plasma D-dimer quartiles (Table 1). Estimated 1-year mortality rates were $42.7 \%$, $76.0 \%, 83.9 \%$, and $86.3 \%$ in patients with $1 \mathrm{st}, 2 \mathrm{nd}$, $3 \mathrm{rd}$, and 4 th D-dimer quartiles, respectively.

\section{Hypercoagulability and other predictors of survival}

Kaplan-Meier analysis showed that higher baseline D-dimer quartiles, cryptogenic stroke mechanism, hepatobiliary-pancreatic cancer type, and systemic metastasis were significantly associated with poor survival (Log-rank test; all $P<0.001$, Table 2). Quartiles of baseline plasma D-dimer level provided significantly different survival curves, but were not different between 3rd and 4th quartiles for both overall and 1-year survival (both $P>0.999$ by log-rank tests after Bonferroni correction; Figure 2A, B). Therefore, we combined 3rd-4th quartiles together in the Cox regression model. Univariate analysis revealed that high (3rd-4th quartiles) pre-treatment plasma D-dimer levels were associated with increased mortality (HR 3.08, 95\% Cl 2.15-4.40 for overall survival; HR 3.78, 95\% Cl 2.48-5.75 for 1-year survival; Figure 2B). In the final multivariate model, patients with 3rd-4th D-dimer quartiles have more than two-fold higher hazard rate than those in the lowest quartile for overall and 1-year survival (adjusted $\mathrm{HR}, 2.19$ [95\% Cl, 1.46-3.31] and 2.70 [95\% Cl, 1.68-4.35], both $P<0.001$; Figure $2 \mathrm{C}$ ). The complete results of predefined predictors from Cox regression models are presented in the online Supplementary Table 1. The result remained significant in subgroups stratified by the presence of systemic metastasis and by stroke mechanisms (cryptogenic vs conventional mechanism) (see online Supplementary Table 2).

\section{Correction of coagulopathy and survival}

Among the 132 patients with high D-dimer levels (3rd-4th quartiles), 113 (85.6\%) underwent anticoagulation therapy. A minority of the patients $(n=19)$ did not undergo anticoagulation because of bleeding diathesis $(n=9)$ or severe stroke $(n=10)$. In 113 patients who received anticoagulation treatment, the posttreatment D-dimer levels were sampled at a median of 7 (IOR 6-7) days after anticoagulation. Plasma D-dimer levels significantly reduced after treatment (mean difference: $-16.22 \mu \mathrm{g} / \mathrm{mL}$, 95\% $\mathrm{Cl},-13.00--19.43, P<0.001$, paired t-test). The post-treatment D-dimer level was median 8.17 (IOR 3.70-14.21, range $0.46-55.18) \mu \mathrm{g} / \mathrm{mL}$. In univariate Cox regression analysis, posttreatment D-dimer levels were significantly associated with both overall and 1-year survival (HR 1.02; $95 \% \mathrm{Cl}, 1.00-1.04$ per 1 $\mu \mathrm{g} / \mathrm{mL}$ increase, $P=0.019$ for overall survival; $\mathrm{HR} 1.03 ; 95 \% \mathrm{Cl}$, 1.01-1.04 per $1 \mu \mathrm{g} / \mathrm{mL}$ increase, $P=0.004$ for 1 -year survival). Multivariate analysis revealed that the post-treatment $\mathrm{D}$-dimer level was independently associated with poor 1-year survival (adjusted HR 1.03; 95\% Cl, 1.01-1.05 per $1 \mu \mathrm{g} / \mathrm{mL}$ increase, $P=$ 0.015 ) but not with overall survival. Pre-treatment $D$-dimer level was no longer significant in association with survival in this subgroup. Rather, there was a significant interaction between pretreatment and post-treatment D-dimer level on survival, whereby the impact of post-treatment D-dimer level on survival was moderated by the pre-treatment level with a negative coefficient (unstandardized beta $=-0.002, \mathrm{SE}=0.001, P$ for interaction $=$ 0.001 for both overall and 1-year survival).

Successful correction of hypercoagulability $(<3 \mu \mathrm{g} / \mathrm{mL})$ was achieved in 19 (16.8\%) patients. Survival curves were clearly di- 
Table 1. Patient characteristics

\begin{tabular}{|c|c|c|c|c|c|c|}
\hline & \multirow{2}{*}{$\begin{array}{l}\text { Total patients } \\
\qquad(\mathrm{N}=268)\end{array}$} & \multicolumn{5}{|c|}{ D-dimer quartiles (range, $\mu \mathrm{g} / \mathrm{mL})^{*}$} \\
\hline & & $\begin{array}{c}1 \text { st }(<2.08) \\
n=66\end{array}$ & $\begin{array}{l}\text { 2nd (2.08-9.06) } \\
\qquad n=65\end{array}$ & $\begin{array}{c}3 r d(9.06-23.26) \\
\quad n=67\end{array}$ & $\begin{array}{c}\text { 4th }(>23.26) \\
n=65\end{array}$ & $P$ \\
\hline \multicolumn{7}{|c|}{ Demographics and stroke characteristics } \\
\hline Age (year) & $66(58-73)$ & 69 (59-73) & $66(57-75)$ & $65(56-71)$ & $66(60-73)$ & 0.454 \\
\hline Male sex $(\%)$ & $159(59.3)$ & $49(74.2)$ & $43(66.2)$ & $25(39.1)$ & $35(53.8)$ & 0.001 \\
\hline Hypertension (\%) & $101(37.7)$ & $29(43.9)$ & $26(40.0)$ & $21(32.8)$ & $22(33.8)$ & 0.512 \\
\hline Diabetes $(\%)$ & $55(20.5)$ & $15(22.7)$ & $15(23.1)$ & $13(20.3)$ & $10(15.4)$ & 0.679 \\
\hline Dyslipidemia (\%) & $24(9.0)$ & $13(19.7)$ & 4 (6.2) & $0(0.0)$ & $5(7.7)$ & 0.001 \\
\hline Coronary artery disease (\%) & $40(14.9)$ & $13(19.7)$ & $9(13.8)$ & $10(15.6)$ & $6(9.2)$ & 0.398 \\
\hline Atrial fibrillation (\%) & $22(8.2)$ & $8(12.1)$ & $6(9.2)$ & $4(6.3)$ & $2(3.1)$ & 0.243 \\
\hline Smoking (\%) & $57(21.3)$ & $20(30.3)$ & $14(21.5)$ & $10(15.6)$ & $10(15.4)$ & 0.119 \\
\hline NIHSS & $4(2-9)$ & $3(1-7)$ & $4(2-9)$ & $5(2-8)$ & $5(1-15)$ & 0.442 \\
\hline \multicolumn{7}{|l|}{ Stroke mechanism } \\
\hline Cryptogenic (\%) & 192 (71.6) & $23(34.8)$ & $50(76.9)$ & $54(84.4)$ & $62(95.4)$ & $<0.001$ \\
\hline Conventional (\%) & & & & & & 0.104 \\
\hline LAA & $33(12.3)$ & $20(46.5)$ & $8(53.3)$ & $2(20.0)$ & $1(33.3)$ & \\
\hline SAO & $14(5.2)$ & $12(27.9)$ & $0(0.0)$ & $1(10.0)$ & $0(0.0)$ & \\
\hline Cardioembolism & $28(10.4)$ & $10(23.3)$ & $7(46.7)$ & $7(70.0)$ & $2(66.7)$ & \\
\hline Other & $1(0.4)$ & $1(2.3)$ & $0(0.0)$ & $0(0.0)$ & $0(0.0)$ & \\
\hline \multicolumn{7}{|l|}{ Pre-stroke medication } \\
\hline Anticoagulant & $15(5.6 \%)$ & $3(4.5 \%)$ & $4(6.2 \%)$ & $5(7.5 \%)$ & $3(4.6 \%)$ & 0.923 \\
\hline Antiplatelet agent & $18(6.7 \%)$ & $8(12.1 \%)$ & $2(3.1 \%)$ & $3(4.5 \%)$ & $5(7.7 \%)$ & 0.203 \\
\hline \multicolumn{7}{|l|}{ Coagulation Markers } \\
\hline D-dimer ( $\mu \mathrm{g} / \mathrm{mL}$ ) (range) & $9.06(0.22-60)$ & $0.92(0.22-2.08)$ & $3.51(2.18-8.72)$ & 15.58 (9.06-23.26) & $38.9(23.3-60)$ & $<0.001$ \\
\hline PT (second) & $13.4(12.9-14.2)$ & $13.9(12.8-15.2)$ & $14.4(13.2-16.1)$ & 15.8 (15.0-16.8) & $13.4(12.9-14.2)$ & $<0.001$ \\
\hline aPTा (second) & $36.3(32.2-42.3)$ & $35.3(31.5-43.1)$ & 33.7 (31.3-39.3) & $36.3(33.3-41.0)$ & $36.3(32.2-42.3)$ & 0.135 \\
\hline Fibrinogen (mg/dL) & $399(319-536)$ & $421(282-515)$ & 319 (195-419) & $215(152-333)$ & $399(319-536)$ & $<0.001$ \\
\hline Platelet $\left(\times 10^{3} / \mu \mathrm{L}\right)$ & $225(176-326)$ & $170(122-247)$ & 149 (87-218) & 122 (85-188) & $225(176-326)$ & $<0.001$ \\
\hline \multicolumn{7}{|l|}{ Cancer characteristics } \\
\hline Cancer type & & & & & & 0.038 \\
\hline Stomach/esophagus (\%) & 32 (11.9) & $11(16.7)$ & $8(12.3)$ & $7(10.9)$ & $5(7.7)$ & \\
\hline Pancreatic (\%) & $19(7.1)$ & $2(3.0)$ & $4(6.2)$ & $5(7.8)$ & $8(12.3)$ & \\
\hline Colorectal (\%) & $20(7.5)$ & $8(12.1)$ & $3(4.6)$ & $3(4.7)$ & $5(7.7)$ & \\
\hline Urological (\%) & $14(5.2)$ & $6(9.1)$ & $2(3.1)$ & $3(4.7)$ & $2(3.1)$ & \\
\hline Hepatobiliary (\%) & 31 (11.6) & $3(4.5)$ & $14(21.5)$ & $6(9.4)$ & $8(12.3)$ & \\
\hline Lung (\%) & 105 (39.2) & $25(37.9)$ & $25(38.5)$ & $25(39.1)$ & $26(40.0)$ & \\
\hline Gynecologic (\%) & $21(7.8)$ & $2(3.0)$ & $3(4.6)$ & $11(17.2)$ & $5(7.7)$ & \\
\hline Breast (\%) & $4(1.5)$ & $2(3.0)$ & $0(0.0)$ & $2(3.1)$ & $0(0.0)$ & \\
\hline Others (\%) & $22(8.2)$ & 7 (10.6) & $6(9.2)$ & $2(3.1)$ & $6(9.2)$ & \\
\hline Adenocarcinoma (\%) & $178(66.4)$ & $40(62.5)$ & $38(64.4)$ & 48 (78.7) & 47 (78.3) & 0.079 \\
\hline Systemic metastasis (\%) & $176(65.7)$ & $30(48.4)$ & $37(60.7)$ & $54(85.7)$ & 55 (85.9) & $<0.001$ \\
\hline Stroke treatment & & & & & & $<0.001$ \\
\hline No treatment (\%) & $20(7.5)$ & $0(0.0)$ & $6(9.2)$ & $5(7.8)$ & $8(12.3)$ & \\
\hline Antiplatelet agent (\%) & 51 (19.0) & 27 (40.9) & $12(18.5)$ & $3(4.7)$ & $2(3.1)$ & \\
\hline Anticoagulation (\%) & $203(75.7)$ & 39 (59.1) & 47 (72.3) & $56(87.5)$ & $55(84.6)$ & \\
\hline Heparin/warfarin (\%) & $113(42.2)$ & $33(50.0)$ & $29(44.6)$ & $24(37.5)$ & 22 (33.8) & \\
\hline LMWH (\%) & 89 (33.2) & $6(9.1)$ & $18(27.7)$ & $32(50.0)$ & $33(50.8)$ & \\
\hline
\end{tabular}

Values are median (interquartile range), unless otherwise specified.

NIHSS, National Institutes of Health Stroke Scale; LAA, large-artery atherosclerosis; SAO, small artery occlusion; LMWH, low-molecular-weight heparin.

*Five patients whose coagulation markers were not sampled per protocol were excluded.

vided by the achievement of successful correction of hypercoagulability (log-rank $P=0.011$ for overall survival and 0.003 for 1-year survival; Figure $3 A, B)$. In the multivariate Cox survival analysis, the successful correction of hypercoagulability was a protective factor for 1-year survival (adjusted HR 0.26 [Cl 0.100.68 ], $P=0.006$; Figure $3 \mathrm{C}$ ), not for overall survival. Hepatobiliary-pancreatic cancers and atrial fibrillation were also significant for 1-year survival after stroke (Figure $3 \mathrm{C}$ ). Other covariates in- 
Table 2. Overall survival of patients with stroke and active cancer by predefined prognostic factors

\begin{tabular}{|c|c|c|c|c|}
\hline & \multicolumn{4}{|c|}{ Total patients $(\mathrm{N}=268)$} \\
\hline & No. (\%) & $\begin{array}{c}\text { Median survival, days } \\
\text { (95\% Cl) }\end{array}$ & $P$ value ${ }^{*}$ & $P$ value $^{\dagger}$ \\
\hline Baseline D-dimer quartiles & & & $<0.001$ & \\
\hline $1 \mathrm{st}(<2.08 \mu \mathrm{g} / \mathrm{mL})$ & $66(25.1)$ & 440 (306 to 574$)$ & & $<0.001$ \\
\hline 2nd (2.08-9.06 $\mu \mathrm{g} / \mathrm{mL})$ & $65(24.7)$ & 162 (112 to 212$)$ & & 0.044 \\
\hline $3 \mathrm{rd}(9.06-23.26 \mu \mathrm{g} / \mathrm{mL})$ & $67(25.5)$ & 64 (39 to 89) & & $>0.999$ \\
\hline 4th $(>23.26 \mu \mathrm{g} / \mathrm{mL})$ & $65(24.7)$ & 66 (43 to 89) & & (Ref) \\
\hline Age stratified by median & & & 0.681 & \\
\hline$<66$ years & $127(47.4)$ & 100 (59 to 141$)$ & & \\
\hline$\geq 66$ years & $141(52.6)$ & 122 (79 to 165$)$ & & \\
\hline Atrial fibrillation & & & 0.689 & \\
\hline Yes & $22(8.2)$ & 174 (71 to 277$)$ & & \\
\hline No & $246(91.7)$ & 107 (82 to 132$)$ & & \\
\hline NIHSS stratified by median & & & 0.775 & \\
\hline$<4$ & $108(40.3)$ & 109 (70 to 148$)$ & & \\
\hline$\geq 4$ & $130(48.6)$ & 105 (67 to 143$)$ & & \\
\hline Stroke mechanism & & & $<0.001$ & \\
\hline Cryptogenic & 192 (71.6) & 82 (62 to 102) & & \\
\hline Conventional mechanism & & 297 (179 to 415$)$ & & \\
\hline Cancer type & & & $<0.001$ & \\
\hline Hepatobiliary/pancreas & $50(18.7)$ & 67 (61 to 73) & & \\
\hline UGI/LGI & $52(19.4)$ & 122 (0 to 293) & & \\
\hline Lung & $105(39.2)$ & 167 (95 to 239) & & \\
\hline Others & $61(22.8)$ & $130(40$ to 220$)$ & & \\
\hline Adenocarcinoma & & & 0.409 & \\
\hline Yes & $178(66.4)$ & 130 (83 to 177 ) & & \\
\hline No & $74(29.4)$ & 94 (47 to 141$)$ & & \\
\hline Systemic metastasis & & & $<0.001$ & \\
\hline Yes & $176(65.7)$ & 78 (61 to 95) & & \\
\hline No & $82(46.6)$ & 535 (253 to 817$)$ & & \\
\hline
\end{tabular}

$\mathrm{Cl}$, confidence interval; NIHSS, National Institutes of Health Stroke Scale; UGI, upper gastrointestinal; LGI, lower gastrointestinal.

*by Log-rank tests pooled over strata; ${ }^{+}$by the Log-rank test compared with 4th quartile, with Bonferroni correction for multiple comparisons.

cluding baseline D-dimer concentrations, systemic metastasis, cryptogenic stroke mechanism, age, stroke severity, adenocarcinoma histology, platelet count, prothrombin time, and fibrinogen level were not significantly associated with survival. The baseline D-dimer concentrations and intervals of D-dimer sampling were not significant to the outcomes.

After discharge, plasma D-dimer levels were followed up at the outpatient neurology clinic in 136 patients. The plasma Ddimer level was significantly associated with death within 1 month (OR 1.07; 95\% Cl, 1.04-1.10 per $1 \mu \mathrm{g} / \mathrm{mL}$ increase, $P<0.001)$. The ROC curve analysis revealed that the plasma Ddimer levels at any time was a significant predictor of death within the next month (area under the curve $=0.835\left[95 \% \mathrm{Cl}_{\text {, }}\right.$ 0.784 to 0.886$], P<0.001$ ) with an optimal cutoff of $3.17 \mu \mathrm{g} / \mathrm{mL}$ (Figure 4).

\section{Discussion}

The major finding of the present study is that cancer-related hypercoagulability, as measured by plasma D-dimer levels, is associated with decreased survival in patients with stroke and active cancer. In patients with severe hypercoagulability, posttreatment plasma D-dimer level further divided survival curves within 1 year after stroke. Outside the acute stroke setting, higher plasma D-dimer levels were predictive of death within the next month of measurement.

Stroke in patients with cancer has distinctive features that differ from those of conventional stroke and also has a higher mortality. ${ }^{1}$ In the present study, the mortality rate was $18.3 \%$ at 1 month and $71.6 \%$ at 1 year. Early mortality was driven mostly by patients with severe hypercoagulability, who had less conventional risk factors of stroke outcome. However, those patients also had more widespread metastasis and aggressive cancer types than patients without hypercoagulability. Therefore, it is both 

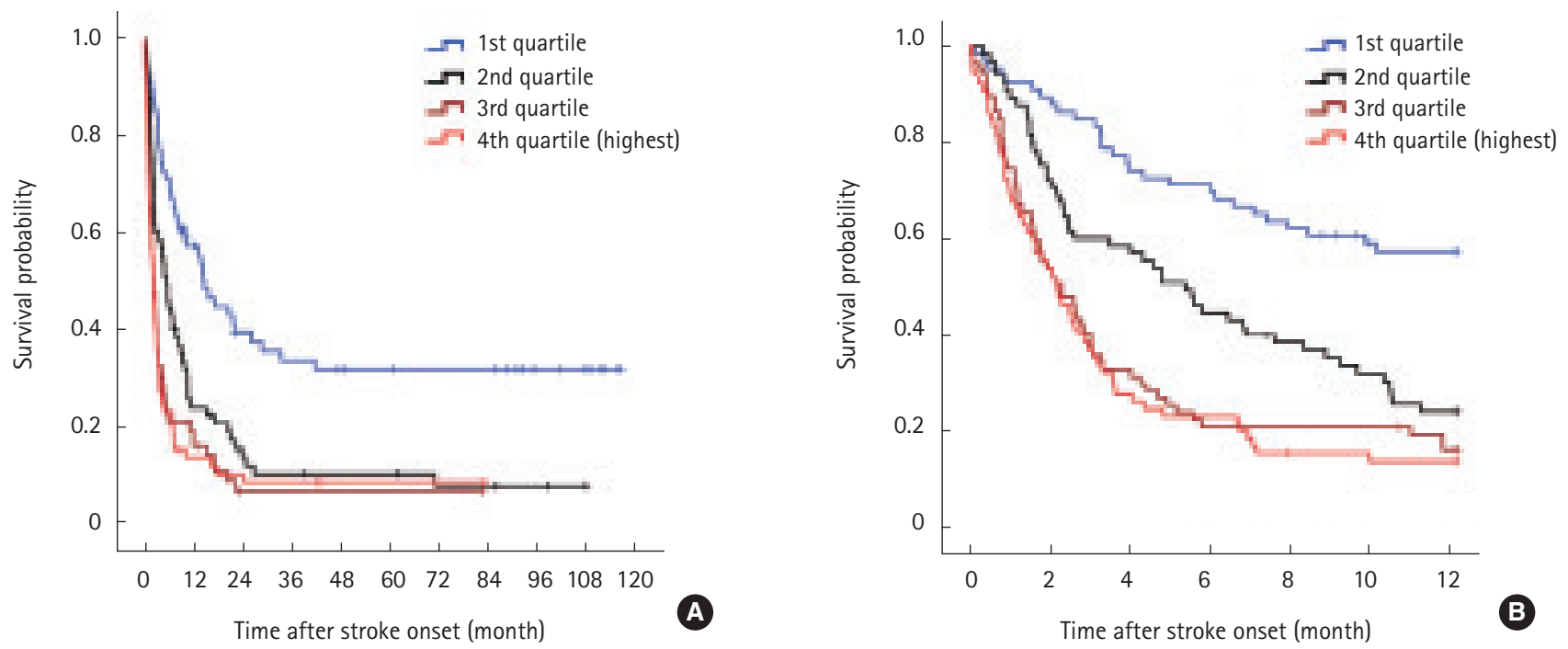

\begin{tabular}{|c|c|c|c|c|}
\hline & \multicolumn{2}{|l|}{ Overall mortality } & \multicolumn{2}{|l|}{ 1-year mortality } \\
\hline & $\begin{array}{c}\text { Univariate } \mathrm{HR} \\
(95 \% \mathrm{CI})\end{array}$ & $\begin{array}{c}\text { Multivariate } \mathrm{HR} \\
(95 \% \mathrm{Cl})\end{array}$ & $\begin{array}{c}\text { Univariate } \mathrm{HR} \\
(95 \% \mathrm{Cl})\end{array}$ & $\begin{array}{c}\text { Multivariate } \mathrm{HR} \\
(95 \% \mathrm{Cl})\end{array}$ \\
\hline \multicolumn{5}{|l|}{$\begin{array}{l}\text { Pretreatment Plasma } \\
\text { D-dimer level (quartiles) }\end{array}$} \\
\hline 1st $(<2.08 \mu \mathrm{g} / \mathrm{mL})$ & (Ref) & (Ref) & (Ref) & (Ref) \\
\hline 2nd (2.08-9.06 $\mu \mathrm{g} / \mathrm{mL})$ & $2.01(1.35-2.99)^{\star}$ & $1.44(0.92-2.26)$ & $2.30(1.45-3.67)^{*}$ & $1.62(0.95-2.76)$ \\
\hline 3rd-4th $(>9.06 \mu \mathrm{g} / \mathrm{mL})$ & $3.08(2.15-4.40)^{\star}$ & $2.19(1.46-3.31)^{\star}$ & $3.78(2.49-5.75)^{*}$ & $2.70(1.68-4.35)^{\star}$ \\
\hline Systemic metastasis & $3.51(2.51-4.91)^{*}$ & $2.91(1.95-4.34)^{*}$ & $3.48(2.39-5.07)^{*}$ & $2.96(1.86-4.70)^{*}$ \\
\hline $\begin{array}{l}\text { Hepatobiliary/pancreatic } \\
\text { cancer }\end{array}$ & $2.01(1.45-2.77)^{*}$ & $2.25(1.54-3.29)^{*}$ & $2.08(1.49-2.90)^{*}$ & $2.23(1.51-3.28)^{*}$ \\
\hline NIHSS (per 1 point increase) & $1.03(1.01-1.06)^{*}$ & $1.03(1.01-1.06)^{*}$ & $1.04(1.01-1.06)^{\star}$ & $1.03(1.00-1.06)^{\star}$ \\
\hline
\end{tabular}

Figure 2. Kaplan-Meier survival curves by quartiles of pre-treatment plasma D-dimer levels (A) during the study period and (B) within 1 year after stroke (Log-rank test $P<0.001$ for both). (C) Univariate and multivariate Cox regression analysis for overall and 1-year survival after stroke. Age, systemic metastasis, NIHSS, primary cancer type (hepatobiliary-pancreatic vs others), atrial fibrillation, adenocarcinoma histology, and stroke mechanism (cryptogenic vs. conventional) were adjusted for in the multivariate model using a forward stepwise method. Only significant variables in each multivariate model are shown in the Table. ${ }^{*} P<0.05$.

possible that hypercoagulability itself might be the leading cause of death, or a marker of more fatal nature of the cancer itself.

The association between cancer and increased thrombosis is well known. The tumor cell promotes a hypercoagulable state and activates the clotting cascade via tumor procoagulants such as tissue factors, cancer procoagulants, and tumor mucins. ${ }^{18}$ Conversely, preclinical studies have shown that hypercoagulability can precipitate tumor growth or metastasis progression. ${ }^{18,19}$ As a consequence of the bidirectional causal relationship, venous thromboembolism negatively affects survival in patients with cancer. ${ }^{20,21}$ Similarly, hypercoagulability may play a role in survival after stroke in patients with cancer.,

In this study, plasma D-dimer concentration, a surrogate marker of hypercoagulability, was an independent predictor of overall and 1-year survival after stroke in patients with cancer.
Our study subjects had a high level of pre-treatment D-dimer levels with a median of $9.06 \mu \mathrm{g} / \mathrm{mL}$, which is much higher than the levels observed in patients who had non-cancer strokes ${ }^{22}$ and even large infarctions. ${ }^{23} \mathrm{D}$-dimer, a stable end-product of fibrin degradation, is a direct measure of activated coagulation. ${ }^{1}$ The plasma D-dimer level is a sensitive marker of cancer-associated hypercoagulable state. ${ }^{1,4,6,7,24}$ We previously reported the significance of the plasma D-dimer level as a marker for diagnosis and treatment response in cancer-related stroke. ${ }^{3,7,25}$ Extremely increased coagulation might also be associated with consumptive coagulopathy. Thus, it is unlikely that the association between mortality and high plasma D-dimer concentration is mediated by disseminated intravascular coagulopathy (DIC). In our multivariate model, post-treatment plasma D-dimer concentration was a significant predictor of death after controlling for DIC compo- 

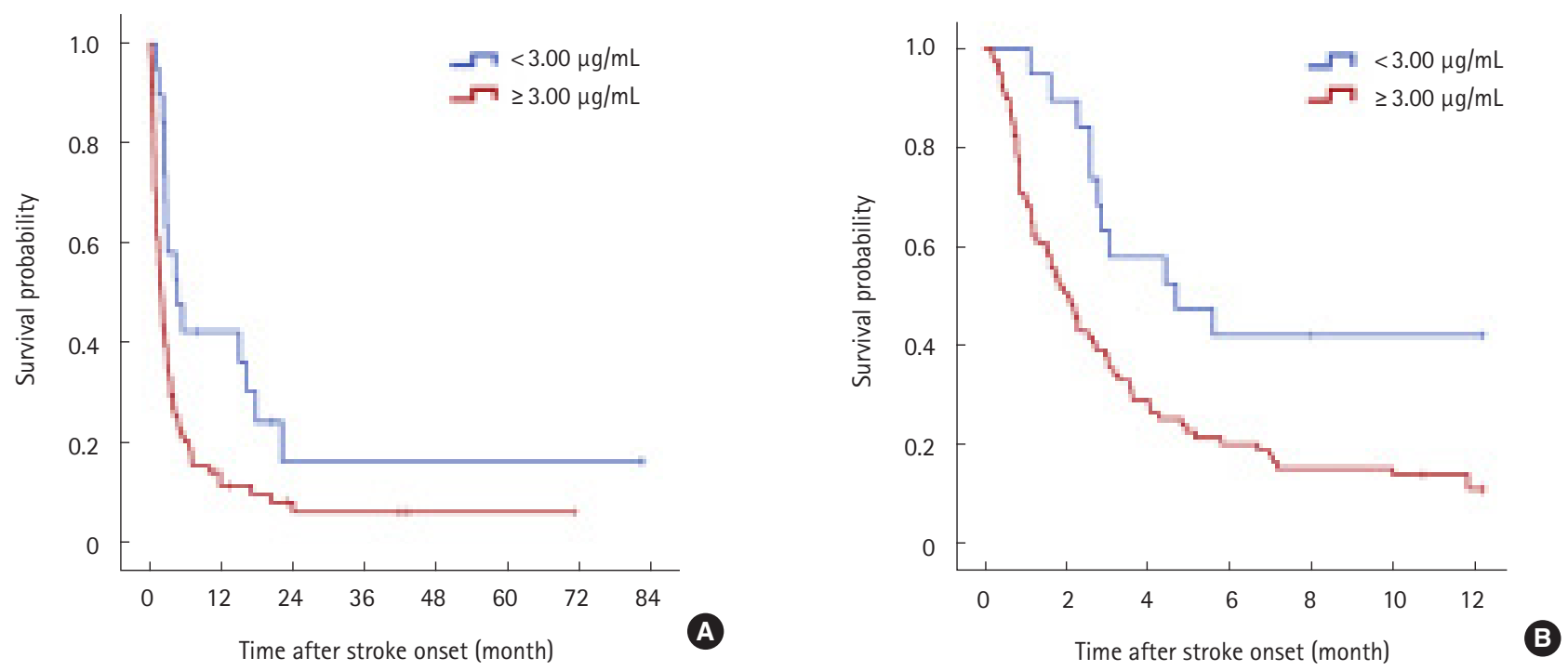

\begin{tabular}{lcccc}
\hline & \multicolumn{3}{c}{ Overall mortality } & 1-year mortality \\
\cline { 2 - 5 } & $\begin{array}{c}\text { Univariate HR } \\
(95 \% \mathrm{Cl})\end{array}$ & $\begin{array}{c}\text { Multivariate HR } \\
(95 \% \mathrm{Cl})\end{array}$ & $\begin{array}{c}\text { Univariate } \mathrm{HR} \\
(95 \% \mathrm{Cl})\end{array}$ & $\begin{array}{c}\text { Multivariate HR } \\
(95 \% \mathrm{Cl})\end{array}$ \\
\hline $\begin{array}{l}\text { Post-treatment D-dimer level } \\
<3.00 \mu \mathrm{g} / \mathrm{mL}\end{array}$ & $0.49(0.28-0.86)^{*}$ & N.S. & $0.40(0.21-0.75)^{*}$ & $0.26(0.10-0.68)^{*}$ \\
$\begin{array}{l}\text { Hepatobiliary/pancreatic } \\
\text { cancer }\end{array}$ & $2.12(1.29-3.46)^{*}$ & $2.53(1.37-4.67)^{*}$ & $2.12(1.29-3.46)$ & $2.45(1.32-4.55)^{*}$ \\
Atrial fibrillation & $1.34(0.58-3.07)$ & N.S. & $1.34(0.58-3.07)$ & $5.98(1.42-25.23)^{*}$ \\
\hline
\end{tabular}

Figure 3. Kaplan-Meier survival curves (A) during the study period and (B) within 1 year after stroke by the achievement of successful correction of hypercoagulable states (D-dimer levels $<3 \mu \mathrm{g} / \mathrm{mL}$ after anticoagulation) in patients with high baseline D-dimer levels (3rd and 4th quartiles) who underwent anticoagulation treatment (Log-rank $P=0.011$ and 0.003 , respectively). Post-treatment plasma D-dimer levels were determined at 7 days after anticoagulation treatment. (C) Univariate and multivariate Cox regression analysis for overall and 1-year survival after stroke. Age, thrombocytopenia ( $>100,50-100,<50 \times 10^{3} / \mu \mathrm{L}$ ), hypofibrinogenaemia ( $>100 \mathrm{vs}$. $<100 \mathrm{mg} / \mathrm{dL}$ ), prothrombin time ( 3 vs. $>3$ seconds), baseline plasma Ddimer concentrations, systemic metastasis, NIHSS, primary cancer type (hepatobiliary-pancreatic vs others), atrial fibrillation, adenocarcinoma histology, and stroke mechanism (cryptogenic vs. conventional) were adjusted for in the multivariate model using a forward stepwise method. Only significant variables in each multivariate model are shown in the Table. ${ }^{*} P<0.05$.

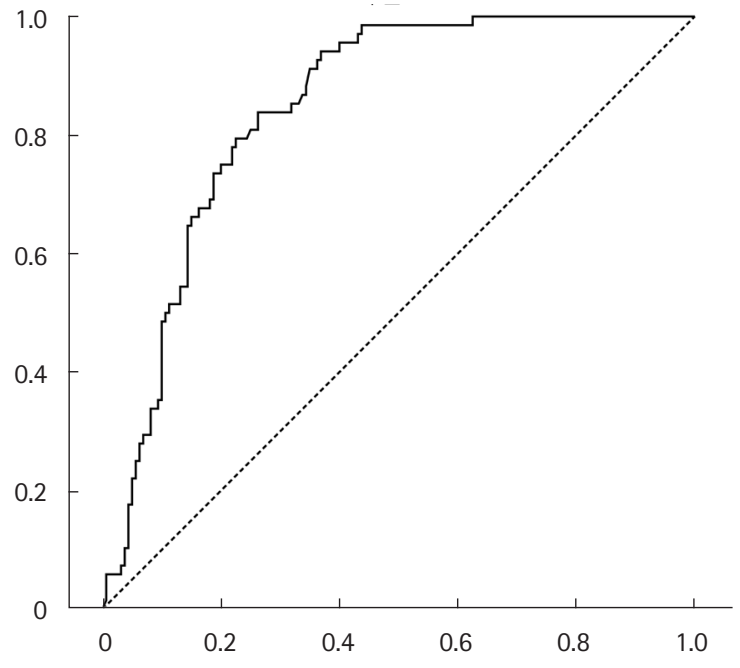

Figure 4. The receiver operating characteristic ROC curve of plasma Ddimer levels measured at the outpatient clinic to predict death within 1 month (area under the curve $=0.835[95 \% \mathrm{Cl}, 0.784-0.886], P<0.001$ ). nents. ${ }^{16,26}$ In addition, our recent study results showed that the level of circulating cancer cell-derived extracellular vesicles, ${ }^{27}$ which are known to play a role in cancer progression (i.e., carcinogenesis/metastasis, coagulation, and tumor growth), correlated with plasma D-dimer levels. ${ }^{28}$

The plasma D-dimer level after anticoagulation treatment was a significant predictor of 1-year survival in patients with severe hypercoagulability. Moreover, the interaction analysis showed that if the post-treatment D-dimer level is similar in all the patients, those who had higher pre-treatment D-dimer level, which implies marked reduction of D-dimer level after treatment, were more likely to survive. Conversely, those whose D-dimer level was relatively low but increased despite anticoagulation had worse survival outcome. Our finding suggests that the treatment response to anticoagulation is associated with better survival in patients with severe hypercoagulability. Evidence suggests that anticoagulants might prevent cancer progression and improve 
overall survival in patients with cancer. ${ }^{18,29}$ In our study, in the subjects with high rate (85.6\%) of anticoagulation treatment, anticoagulation itself could not be compared with other antithrombotic treatments. Rather, post-treatment D-dimer levels were followed up to assess whether hypercoagulability was effectively corrected. There might be several factors that influence the efficacy of anticoagulation, such as underlying cancer activity, coexistence with DIC, and intensity of anticoagulation treatment, of which the former two were controlled for in the multivariate analysis. Our study results encourage further prospective studies comparing fixed-dose anticoagulation and targeted therapy with careful monitoring of the coagulation status.

Our data imply that traditional prediction models of stroke mortality might not explain outcomes of patients with cancerrelated stroke. Age has been used in various models of outcome prediction in patients with stroke, ${ }^{8,30,31}$ but was not significant in our study. On the other hand, cancer characteristics such as metastasis, primary cancer type, and cancer-related hypercoagulability had a considerable effect on survival in the present study. Contrary to previous reports, ${ }^{1}$ pancreatic and hepatobiliary cancers including cholangiocarcinoma were more unfavorable than lung cancers in our cohort. Hepatobiliary and pancreatic cancers may have an intrinsic fatality as they are often unresectable even in the absence of systemic metastasis. ${ }^{32-34}$ Among lung cancers, adenocarcinomas were most common in our study subjects. Adenocarcinoma has been reported to be related to recurrent thromboembolism, which leads to poor survival in patients with cancer and stroke. ${ }^{35}$ However, our findings suggest that both increased coagulation and primary cancer type determine the prognosis of adenocarcinomas. Atrial fibrillation was not an independent prognostic factor in the entire cancer-stroke cohort in our study, but led to poor 1-year survival in patients having severe hypercoagulability, possibly via an additive effect of slow flow and increased coagulation.

After the acute stroke setting, high plasma D-dimer concentrations measured in the outpatient clinic were associated with death within the next month. This could not be explained by stroke severity or consumptive coagulopathies. Instead, it is likely that increased coagulation can be a marker of increased cancer activity, effectiveness of antithrombotic treatment, or both. Although the causal inference could not be made in this observational study, data suggest that hypercoagulable states, if not effectively controlled, may lead to cancer progression via the action of thrombin on adhesion, mitosis, and angiogenesis in tumor cells. ${ }^{19}$ Thus, monitoring of coagulation markers may be helpful even after recovery from stroke.

The strengths of this study include the large number of samples, a prospective collection of data, comprehensive and ho- mogenous evaluations in a single tertiary center, long-term follow-up data during and after hospitalization, and high rates of survival identification. However, our study is not without limitations. First, cancer treatment was not included as a predictor of survival, because anticancer treatment is influenced by limited life expectancy, which can lead to a self-fulfilling bias. ${ }^{36}$ Furthermore, both acute stroke and consequent functional impairment are often regarded as a contraindication of active anti-cancer treatment. A different strategy should be established to determine the optimal anti-cancer treatment in stroke survivors. Second, antithrombotic treatment was not controlled for in this observational study. However, most of the patients with severe hypercoagulability underwent anticoagulation treatment. We analyzed the post-treatment D-dimer levels only in subgroups that underwent anticoagulation treatment to minimize ascertainment bias. Third, co-existing deep venous thrombosis was not routinely evaluated during the entire study period. Venous thrombosis is a potential cause of increased D-dimer levels and ischemic stroke in the presence of right-to-left shunt. ${ }^{37}$ However, our previous study demonstrated increased intra-arterial thrombosis and its association with increased D-dimer levels in cancerrelated stroke.7 We are conducting a prospective study to investigate the prevalence of deep venous thrombosis and right-toleft shunting in cancer-related stroke (clinicaltrials.gov identifier No. NCT02212496). Fourth, we did not determine the cause of death. Death in cancer patients can be attributed to cancer itself, as well as complications such as infection or thromboembolic events. Further research should be performed to reveal how increased coagulation affects survival. Finally, the data were evaluated from a single population. Our study subjects had a higher proportion of cryptogenic stroke than in previous studies. ${ }^{9}$ This might be related, in part, to increased detection of hidden active cancer in cryptogenic stroke with hypercoagulable states in our center. ${ }^{3}$ Further investigations of different study populations are needed before generalization of our results.

\section{Conclusion}

In conclusion, hypercoagulability is associated with poor survival in cancer-stroke, warranting monitoring and effective anticoagulation treatment for better survival. Plasma D-dimer levels might reflect the severity of the hypercoagulable states, treatment response, and risk of subsequent death in both acute hospitalized and chronic outpatient settings. Large-scale randomized prospective trials are needed to further evaluate the role of effective correction of hypercoagulability on survival after stroke in patients with active cancer. 


\section{Acknowledgements}

The authors thank all the patients who participated in the study.

\section{References}

1. Cestari DM, Weine DM, Panageas KS, Segal AZ, DeAngelis LM. Stroke in patients with cancer: incidence and etiology. Neurology 2004;62:2025-2030.

2. Navi BB, Reiner AS, Kamel $H$, ladecola C, Elkind MS, Panageas $\mathrm{KS}$, et al. Association between incident cancer and subsequent stroke. Ann Neurol 2015;77:291-300.

3. Kim SJ, Park JH, Lee MJ, Park YG, Ahn MJ, Bang OY. Clues to occult cancer in patients with ischemic stroke. PLOS ONE 2012; 7:e44959.

4. Kim SG, Hong JM, Kim HY, Lee J, Chung PW, Park KY, et al. Ischemic stroke in cancer patients with and without conventional mechanisms: a multicenter study in Korea. Stroke 2010; 41:798-801.

5. Bang OY, Seok JM, Kim SG, Hong JM, Kim HY, Lee J, et al. Ischemic stroke and cancer: stroke severely impacts cancer patients, while cancer increases the number of strokes. J Clin Neurol 2011;7:53-59.

6. Schwarzbach CJ, Schaefer A, Ebert A, Held V, Bolognese M, Kablau $M$, et al. Stroke and cancer: the importance of cancerassociated hypercoagulation as a possible stroke etiology. Stroke 2012;43:3029-3034.

7. Seok JM, Kim SG, Kim JW, Chung CS, Kim GM, Lee KH, et al. Coagulopathy and embolic signal in cancer patients with ischemic stroke. Ann Neurol 2010;68:213-219.

8. Smith EE, Shobha N, Dai D, Olson DM, Reeves MJ, Saver JL, et al. Risk score for in-hospital ischemic stroke mortality derived and validated within the Get With the Guidelines-Stroke Program. Circulation 2010;122:1496-1504.

9. Navi BB, Singer $S$, Merkler AE, Cheng NT, Stone JB, Kamel $H$, et al. Cryptogenic subtype predicts reduced survival among cancer patients with ischemic stroke. Stroke 2014;45:2292-2297.

10. Buccheri G, Ferrigno D, Ginardi C, Zuliani C. Haemostatic abnormalities in lung cancer: prognostic implications. Eur J Cancer 1997;33:50-55.

11. Lee AY, Levine MN, Baker RI, Bowden C, Kakkar AK, Prins M, et al. Low-molecular-weight heparin versus a coumarin for the prevention of recurrent venous thromboembolism in patients with cancer. N Engl J Med 2003;349:146-153.

12. Vedovati MC, Germini F, Agnelli G, Becattini C. Direct oral anticoagulants in patients with VTE and cancer: a systematic review and meta-analysis. Chest 2015;147:475-483.
13. Agnelli G, Gussoni G, Bianchini C, Verso M, Mandala M, Cavanna $L$, et al. Nadroparin for the prevention of thromboembolic events in ambulatory patients with metastatic or locally advanced solid cancer receiving chemotherapy: a randomised, placebo-controlled, double-blind study. Lancet Oncol 2009;10: 943-949.

14. AkI EA, Kahale L, Barba M, Neumann I, Labedi N, Terrenato I, et al. Anticoagulation for the long-term treatment of venous thromboembolism in patients with cancer. Cochrane Database Syst Rev 2014;7:CD006650.

15. Streiff MB. The National Comprehensive Cancer Center Network (NCCN) guidelines on the management of venous thromboembolism in cancer patients. Thromb Res 2010;125 Suppl 2:S128-133.

16. Toh $\mathrm{CH}$, Hoots WK. The scoring system of the Scientific and Standardisation Committee on Disseminated Intravascular Coagulation of the International Society on Thrombosis and Haemostasis: a 5-year overview. J Thromb Haemost 2007;5:604606.

17. Ebrahim S, Sung J, Song YM, Ferrer RL, Lawlor DA, Davey Smith G. Serum cholesterol, haemorrhagic stroke, ischaemic stroke, and myocardial infarction: Korean national health system prospective cohort study. BMJ 2006;333:22.

18. Kuderer NM, Ortel TL, Francis CW. Impact of venous thromboembolism and anticoagulation on cancer and cancer survival. J Clin Oncol 2009;27:4902-4911.

19. Nierodzik M, Karpatkin S. Hypercoagulability preceding cancer. Does hypercoagulability awaken dormant tumor cells in the host? J Thromb Haemost 2005:3:577-580.

20. Sorensen HT, Mellemkjaer L, Olsen JH, Baron JA. Prognosis of cancers associated with venous thromboembolism. $N$ Engl J Med 2000;343:1846-1850.

21. Chew HK, Wun T, Harvey D, Zhou H, White RH. Incidence of venous thromboembolism and its effect on survival among patients with common cancers. Arch Intern Med 2006;166: 458-464.

22. Ageno W, Finazzi S, Steidl L, Biotti MG, Mera V, Melzi D'Eril G, et al. Plasma measurement of D-dimer levels for the early diagnosis of ischemic stroke subtypes. Arch Intern Med 2002; 162:2589-2593.

23. Park YW, Koh EJ, Choi HY. Correlation between Serum D-Dimer Level and Volume in Acute Ischemic Stroke. J Korean Neurosurg Soc 2011;50:89-94.

24. Kim K, Lee JH. Risk factors and biomarkers of ischemic stroke in cancer patients. J Stroke 2014;16:91-96.

25. Jang H, Lee JJ, Lee MJ, Ryoo S, Yoon CH, Kim GM, et al. Comparison of Enoxaparin and Warfarin for Secondary Prevention of Cancer-Associated Stroke. J Oncol 2015;2015:502089. 
26. Levi $\mathrm{M}$, Toh $\mathrm{CH}$, Thachil J, Watson HG. Guidelines for the diagnosis and management of disseminated intravascular coagulation. British Committee for Standards in Haematology. Br J Haematol 2009;145:24-33.

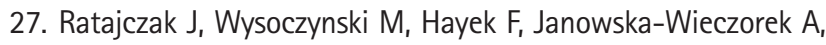
Ratajczak MZ. Membrane-derived microvesicles: important and underappreciated mediators of cell-to-cell communication. Leukemia 2006;20:1487-1495.

28. Bang OY, Chung JW, Lee MJ, Kim SJ, Cho YH, Kim GM, et al. Cancer cell-derived extracellular vesicles are associated with coagulopathy causing ischemic stroke via tissue factor-independent way: the OASIS-CANCER study. PLOS ONE 2016;11: e0159170.

29. Kuderer NM, Khorana AA, Lyman GH, Francis CW. A metaanalysis and systematic review of the efficacy and safety of anticoagulants as cancer treatment: impact on survival and bleeding complications. Cancer 2007;110:1149-1161.

30. Carter AM, Catto AJ, Mansfield MW, Bamford JM, Grant PJ.
Predictive variables for mortality after acute ischemic stroke. Stroke 2007;38:1873-1880.

31. Rabinstein A, Rundek T. Prediction of outcome after ischemic stroke: the value of clinical scores. Neurology 2013;80:15-16.

32. Bruix J, Sherman M. Management of hepatocellular carcinoma: an update. Hepatology 2011;53:1020-1022.

33. Vincent A, Herman J, Schulick R, Hruban RH, Goggins M. Pancreatic cancer. Lancet 2011;378:607-620.

34. Razumilava N, Gores GJ. Cholangiocarcinoma. Lancet 2014; 383:2168-2179.

35. Navi BB, Singer $S$, Merkler AE, Cheng NT, Stone JB, Kamel $H$, et al. Recurrent thromboembolic events after ischemic stroke in patients with cancer. Neurology 2014;83:26-33.

36. Falanga A. Mechanisms of hypercoagulation in malignancy and during chemotherapy. Haemostasis 1998;28 Suppl 3:50-60.

37. Iguchi $Y$, Kimura $K$, Kobayashi $K_{1}$ Ueno $Y$, Inoue T. Ischaemic stroke with malignancy may often be caused by paradoxical embolism. J Neurol Neurosurg Psychiatry 2006;77:1336-1339. 
Supplementary Table 1. Univariate and multivariate Cox proportional hazard models for overall survival

\begin{tabular}{|c|c|c|c|c|}
\hline & \multicolumn{2}{|c|}{ Overall survival } & \multicolumn{2}{|c|}{ 1-year survival } \\
\hline & $\begin{array}{c}\text { Univariate HR } \\
\text { (95\% CI) }\end{array}$ & $\begin{array}{l}\text { Multivariate } \mathrm{HR}^{*} \\
\quad(95 \% \mathrm{Cl})\end{array}$ & $\begin{array}{c}\text { Univariate HR } \\
(95 \% \mathrm{Cl})\end{array}$ & $\begin{array}{c}\text { Multivariate } \mathrm{HR}^{*} \\
\quad(95 \% \mathrm{Cl})\end{array}$ \\
\hline \multicolumn{5}{|l|}{ D-dimer quartiles (range, $\mu \mathrm{g} / \mathrm{mL}$ ) } \\
\hline $01(<2.08)$ & (Ref) & (Ref) & & \\
\hline $02(2.08-9.06)$ & $2.01(1.35-2.99)^{+}$ & $1.44(0.92-2.26)$ & $2.30(1.45-3.67)^{\dagger}$ & $1.62(0.95-2.76)$ \\
\hline 03-04 (>9.06) & $3.08(2.15-4.40)^{+}$ & $2.19(1.46-3.31)^{\ddagger}$ & $3.78(2.49-5.75)^{\dagger}$ & $2.70(1.68-4.35)^{\dagger}$ \\
\hline Systemic metastasis & $3.51(2.51-4.91)^{\dagger}$ & $2.91(1.95-4.34)^{\ddagger}$ & $3.48(2.39-5.07)^{\dagger}$ & $2.96(1.86-4.70)^{\dagger}$ \\
\hline Stroke mechanism ${ }^{\dagger}$ & $1.75(1.30-2.37)^{+}$ & & $1.99(1.41-2.81)^{\ddagger}$ & \\
\hline Age & $1.00(0.99-1.01)$ & & $1.00(0.98-1.01)$ & \\
\hline NIHSS & $1.03(1.01-1.06)^{\ddagger}$ & $1.03(1.01-1.06)^{\dagger}$ & $1.04(1.01-1.06)^{\dagger}$ & $1.03(1.00-1.06)^{\dagger}$ \\
\hline Hepatobiliary/pancreatic cancer & $2.01(1.45-2.77)^{+}$ & $2.25(1.54-3.29)^{\ddagger}$ & $2.08(1.49-2.90)^{\dagger}$ & $2.23(1.51-3.28)^{\dagger}$ \\
\hline Adenocarcinoma & $1.13(0.84-1.52)$ & & $0.87(0.63-1.20)$ & $0.68(0.47-1.00)^{\dagger}$ \\
\hline Atrial fibrillation & $0.91(0.57-1.45)$ & & $0.83(0.49-1.41)$ & \\
\hline
\end{tabular}

${ }^{*}$ Multivariate models adjusted for variables used in univariate analysis with a forward stepwise method; ${ }^{+}$Cryptogenic vs conventional mechanisms (reference); ${ }^{\ddagger} P<0.05$.

$01,02,03$, and 04 denote quartiles. 
Supplementary Table 2. Multivariate Cox proportional hazard models of overall survival in subgroups

\begin{tabular}{|c|c|c|c|c|}
\hline & \multicolumn{2}{|c|}{ Systemic metastasis } & \multicolumn{2}{|c|}{ Stroke mechanism } \\
\hline & Yes & No & Cryptogenic mechanism & Conventional mechanism \\
\hline \multicolumn{5}{|c|}{ D-dimer quartiles (range, $\mu \mathrm{g} / \mathrm{mL}$ ) } \\
\hline $01(<2.08)$ & (Ref) & (Ref) & (Ref) & (Ref) \\
\hline $02(2.08-9.06)$ & $1.47(0.84-2.57)$ & $1.42(0.61-3.30)$ & $1.96(0.94-4.06)$ & $1.19(0.59-2.37)$ \\
\hline $03-04(>9.06)$ & $2.24(1.40-3.60)^{*}$ & $5.70(2.35-13.81)^{*}$ & $2.61(1.30-5.24)^{*}$ & $5.47(2.07-14.44)^{*}$ \\
\hline
\end{tabular}

Values are presented as multivariate hazard ratio (95\% confidence interval).

Multivariate models adjusted for age, systemic metastasis, stroke mechanism, NIHSS, primary cancer type, adenocarcinoma histology, and atrial fibrillation with a forward stepwise method.

*Statistically significant.

$01,02,03$, and 04 denote quartiles. 